\title{
Discursos científicos \\ sobre el dolor cronificado sin-causa-orgánica. Incorporando una mirada de género para resignificar-repolitizar el dolor
}

\author{
Margot Pujal i LlombarT \\ Profesora titular Departamento Psicología Social. U.Autónoma de Barcelona \\ margot.pujal@uab.cat \\ Pilar Albertín CARBó \\ Profesora titular Departamento Psicología. U. de Girona \\ pilar.albertin@udg.edu \\ Enrico Mora Malo \\ Profesor agregado Departamento Sociología. U. Autónoma Barcelona \\ Enrico.mora@uab.cat
}

Recibido: 21-05-2014

Aceptado: 10-06-2015

\section{Resumen}

El dolor cronificado sin-causa-orgánica (DC) conocido en terminología psicomédica como fibromialgia (FM) afecta cada vez más a una proporción considerable de mujeres diagnosticadas en sociedades capitalistas. El interés del siguiente artículo es profundizar en la construcción de este síntoma, su tratamiento y los efectos psicosociales tanto en el orden social, como en la vida de las personas que lo padecen utilizando los discursos que se vehiculizan en publicaciones de revistas españolas. El resultado ha sido la emergencia de tres posiciones discursivas hegemónicas: la posición "cientifista", la posición "terapéutica de la conformidad" y la posición "económico-legalista". Cada una de ellas tiene una especificidad característica, pero en su conjunto se potencian produciendo efectos como la ausencia de contexto social para explicar la enfermedad, la desconsideración de diferencias de género en el abordaje y tratamiento, la instrumentalización del dolor para legitimar sus prácticas y la sujección de las mujeres al paradigma psicobiomédico. Se propone una resignificación-repolitización del concepto de dolor.

Palabras clave: dolor cronificado; fibromialgia; discurso; posiciones; perspectiva de género. 


\title{
Scientific Discourses on Chronic Pain without Organic Cause. Incorporating Gender Perspective for Pain Resignifyng-repoliticizing
}

\begin{abstract}
Chronic pain, without any organic or physical cause (DC), which in psycho-medical terminology is known as fibromyalgia, (FM), is diagnosed each year to a considerable number of women in capitalistic societies. Our main interest in the following paper is to go in depth in the elaboration of this symptom, its treatment and the psychosocial effects, both in the social order as well as in the lives of the people who suffer from it. Our main goal in the following paper is to look deeper in the elaboration (conceptualization) of this symptom, its treatment and psychological affects, both in the social order as well as in the lives of the people who suffer from it, we are using linked speeches in Spanish magazines publications. The result has been the emergence of three hegemonic discourse positions: One position "scientist", one "therapeutic of the conformity" position and one "economic and legalistic" position. Each of these has a specific feature, but on the whole, is enhanced, producing effects such as the absence of social context to explain the disease; disregard of gender differences in the management and treatment; the instrumentalization of pain to legitimize their practices and the subjection of women to the "psycho-biomedical" paradigm. In that way, a new significance and politicization of the concept of pain is proposed.
\end{abstract}

Keywords: Chronic Pain; Fibromyalgia; Discourse; Position; Gender Perspective.

\section{Referencia normalizada}

Pujal i Llombart, M., P. Albertín Carbó y E. Mora Malo (2015): “Discursos científicos sobre el dolor cronificado sin-causa-orgánica. Incorporando una mirada de género para resignificar-repolitizar el dolor", Política y Sociedad, 52 (3), pp. 921-948.

Sumario: 1. Introducción. 2. El Discurso hegemónico y las posiciones enunciativas. 3. Corpus textual para el análisis. 4. Posiciones enunciativas del discurso biopsicomédico entorno al DC/ FM. 5. Conclusión. 6. Bibliografía. 7. Anexos.

$$
* * *
$$

\section{Agradecimientos}

Beca Tomas Ibáñez. Universidad Autónoma de Barcelona (2010) e Institut Català de les Dones (2010-1011). Ref. U-27/10. 


\section{Introducción}

En las sociedades tecnológicamente desarrolladas y capitalistas cada vez más se conoce el aumento del fenómeno o síndrome de dolor cronificado ${ }^{1}$ sin causa orgánica (DC), denominado en el sistema sanitario como fibromialgia (FM) y que afecta en un 95\% a mujeres (Valverde et al., 2001; Pastor et al., 2003). En España se conoce una prevalencia estimada de $2,4 \%$ en la población adulta, con un ratio mujer/hombre de $20 / 1$ $(4,2 \% / 0,2 \%)$ y un tiempo transcurrido hasta que llega el diagnóstico de 9,2 años (Ubago et al., 2005). A pesar de ello, se ha observado desigualdades de género en las estrategias terapéuticas, manifestadas por una mayor proporción de hombres diagnosticados de FM en situación de baja laboral (Ruiz et al., 2007). Por otra parte, este síndrome de dolor sin causa orgánica se asocia a otros malestares de expresión emocional también en aumento, como ansiedad y depresión, constituyendo un conjunto de síntomas somáticos-emocionales destacables.

La FM tiene una afectación en las funciones individuales (emocionales, cognitivas y comportamentales) y también en las funciones familiares, laborales y sociales de la persona, ya que resulta inhabilitante en las personas afectadas entre el $25 \%$ y el $50 \%$ (Rivera, 2009). ${ }^{2}$ Por otra parte, junto con las dificultades actuales existentes en la evaluación y tratamiento implica un alto grado de sufrimiento individual y también familiar (ACAF, 2005), con unos gastos socioeconómicos elevados en consumo sanitario tanto directo como indirecto, por el decremento de la productividad tanto remunerada como no remunerada de la persona afectada, y el incremento de malestares dentro del ámbito familiar, falto de recursos para afrontar el problema.

El siguiente trabajo deriva de una investigación más amplia realizada a partir de diferentes fuentes de información (revisión de artículos científicos, entrevistas individuales y grupales a personas afectadas y diferentes profesionales, fórums de soporte a personas y familias que padecen el problema-enfermedad, así como documentos de normas y protocolos de tratamiento, aunque en el siguiente artículo focalizamos la atención sobre los discursos existentes en publicaciones científico-académicas (españolas y latinoamericanas) y los efectos psicosociales que supone su puesta en circulación.

${ }^{1}$ Hablamos de dolor cronificado en lugar de dolor crónico. El dolor cronificado implica que podría ser que no tuviera una causa orgánica y que no tuviera que cronificarse, mientras que el uso de "crónico" implica continuidad en el tiempo y principalmente, de origen orgánico.

2 Según la OMS (1994) la FM se trata de un síndrome caracterizado por un dolor difuso no articular que afecta a los músculos y que se asocia, típicamente con fatiga crónica persistente, sueño no reparador, rigidez generalizada y que frecuentemente, se acompaña de otros síntomas corporales y psicológicos. Los criterios necesarios para el diagnóstico son: la existencia de un dolor difuso presente, como mínimo, durante 3 meses, la palpación dolorosa en 11 de los 18 puntos sensibles posibles y la exclusión de alteraciones analíticas y radiográficas). 
Nuestro interés se basa en dos razones: por un lado, porque atañe directamente a un problema de salud en el cual el discurso médico-psicológico no encuentra los elementos precisos para su resolución, ni causa, ni tratamiento acertado. Ello hace plantearnos la hipótesis de que tal vez, hay un aspecto relegado en los planes psico-médicos para abordar el problema, y que dicho aspecto relegado tiene que ver con una perspectiva social que se integre a lo bio-psico, a través de la cual podamos dar luz a nuevos elementos que permitan alcanzar una comprensión y una intervención más óptima del fenómeno. La otra razón de nuestro acercamiento tiene que ver con la inquietud que nos produce que sea una "patología" tan típicamente femenina, padecida mayoritariamente por mujeres, y a la vez, tan ignorada en cuanto a esta variable (sexo-genero) por la mayoría de la ciencia médico-psicológica. Se trata de la primera causa de consulta de las mujeres en los centros de atención primaria, y primera causa de abandono de la actividad profesional en España, y que afecta a un 30\% de la población femenina si contamos el dolor difuminado en general (Valls, 2008; Valls-Llovet, 2010). Por tanto, nos lleva a poner en el centro de la cuestión el papel del género, generalmente excluido en el abordaje del problema.

Estas razones nos conducen a analizar el dolor como política ya que es tomado por discursos, por líneas de fuerza y de poder social (tecnologías de poder) para configurar realidades cotidianas, identidades, prácticas sociales normativas, a través de diagnósticos, de tratamientos, de consejos terapéuticos, de identidades de paciente o enfermo. Es en este sentido en que nos interesa explorar el dolor cronificado y ver las posibilidades de resignificación y de repolitización partiendo de los discursos dominantes, especialmente los discursos médico-psicológicos y contrastarlos con perspectivas de género o feministas.

En la medicina social de la segunda mitad del siglo XVIII, y sobre todo de la primera mitad del siglo XIX, podemos encontrar un fuerte componente "sociológico", donde muchas de las enfermedades se explican por su "etiología social" (Luz, 2011). Fue a partir de la introducción del modelo evolucionista en el s. XIX — basado en el modelo de las Ciencias biológicas y Ciencias naturales - , cuando se produjo un desplazamiento de las Ciencias sociales hacia las ciencias médicas, sin posibilidad real de que las Ciencias sociales pudieran contribuir en igual medida con sus saberes. El modelo evolucionista del S.XIX ha sido la norma a la hora de trabajar en Ciencias sociales aplicadas a la salud (modelos cuantitativos, correlaciónales y de explicación causal). En cambio, no se han incorporado factores culturales, sociales y de estructura social en la explicación de las enfermedades o de los estados de salud, y ha predominado un enfoque de los discursos científicos centrado en la patología, la desviación y su tratamiento y no tanto en la salud y la calidad de vida de las personas (Conrach y Schneider, 1992).

De esta forma, los problemas sociales, fruto de los conflictos y transformaciones se han canalizado a través de las ciencias de la salud y en muchos casos a través de la patologización y medicalización, que han reconducido el problema traducido en malestar a algo exclusivamente individual, personal, íntimo, totalmente descontextualizado, ahistorizado y asexualizado, sin referentes socioculturales (Burin, 1990; Pujal y Mora, 
2009). Es a mitades del S. XX cuando comienza a criticarse las estrategias de medicalización social y el biopoder que operan en la producción de cuerpos y emociones. Filósofos, sociólogos y antropólogos, como Illich (1975), Foucault (1980), Delvecchio et al. (1992), serán quienes pongan en evidencia el proceso y las formas de estructuración del poder sobre la vida individual y social, a partir de las instituciones ligadas al saber y a las prácticas médicas, interpretadas como estrategias de medicalización social o biopoder. Estas estrategias constituyen una política donde el tratamiento de los problemas sociales y de salud, está ligada a intervenciones terapéuticas que constituyen prácticas de normalización, discursos que hablan de "verdad" enunciados por la institución médica y psicológica. El efecto de estas prácticas institucionales comporta una inscripción y una forma de configurarse los cuerpos y la subjetividad de las personas.

La idea de biopoder se respalda y consolida en la división de los papeles de los sexos, el género es la base de la biopolítica (Rodríguez, 1999), en el sentido de que la construcción de hombres y mujeres ha sido diferente. Y esta diferencia, en la construcción de la mujer, se ha caracterizado por hacerla un sujeto carente de deseo, un sujeto "objeto de deseo de otro", y con unas funciones biológicas y maternales. Barker (2005) explica cómo estos discursos han vinculado a las mujeres con la reproducción sin placer y con la patología (histeria, criminalización). Benhabib y Cornell (1987) muestran como la mujer canaliza muchos de sus malestares a través de distintas patologías o de la automedicación, consiguiendo con ayuda médica adaptarse a la situación, pero no subvertirla o cuestionarla. Estas autoras dicen que la ayuda médica no tiene por qué subvertir la situación, y que dicha ayuda tienen una fuerte carga de valores sociales conformistas al sistema asexual y descontextualizado.

Por tanto, se configuran unos ideales normativos del cuerpo marcados por el género, que asignan símbolos, significados, placeres y funciones en las diferentes partes del cuerpo. Esta inscripción del género en los cuerpos y la subjetividad constituye un proceso esencialmente violento que provoca malestar. Pero esta inscripción violenta del género para modelar los cuerpos y la subjetividad no excluyen la capacidad agente y de resistencia de los sujetos. Los ideales de género se construyen y actualizan continuamente en las actuaciones cotidianas, por lo que su reiteración nunca es completa y de la propia repetición emergen posibilidades de cambio y subversión (Foucault, 1980; Butler, 2001). En este sentido, el fenómeno del dolor puede convertirse en una forma de resistencia aunque precaria, al tiempo que es el producto del disciplinamiento del cuerpo y de su sujeción.

Estas ideas constituirán las bases que la teoría social y de género aportan como claves para entender el malestar que experimentan las mujeres que manifiestan "dolor cronificado" (DC/FM). Partiremos de la situación de DC/FM como una posibilidad que nos permita:

Analizar los discursos-posiciones procedentes de la medicina y la psicología o discursos médico-psicológicos procedentes de artículos científicos y los efectos que 
producen sobre las personas (individuo) y la construcción del problema (enfermedad).

Explorar las cuestiones que emergen desde una perspectiva de sexo-género en relación a este problema y cómo son tratadas desde estas producciones científicas, especialmente en cuanto a la organización social de la vida de las personas afectadas y en cuanto a desigualdades producidas en función del sexo-género de esas personas.

El análisis del discurso es transferible tanto a hombres como a mujeres, pero al afectar más a las mujeres, nos ha hecho pensar en incorporar la perspectiva de género o feminista, de manera, que al estudiar la experiencia de mujeres con fibromialgia pensamos que se pueden encontrar elementos psicosociales diferenciadores en relación al sexo-género, elementos que muestran desigualdades en la vida cotidiana, en los roles, en el mandato y la norma social que se asume desde "ser mujer", factores que hemos intentado ver si establecen relación con la fibromialgia o dolor crónico sin causa orgánica.

Para llevar a cabo estos objetivos hemos organizado el texto en los apartados:

a) el concepto de discurso y su localización a través de diferentes posiciones sociales-relacionales o posiciones enunciativas;

b) el corpus textual y la selección de los textos-artículos;

c) el análisis de la información, obteniendo tres posiciones enunciativas, y describiendo de cada una de ellas y sus efectos discursivos: c.1) características; c.2) construcción del problema o dolor crónico; y c.3) construcción del sujeto/a que padece el problema.

\section{El Discurso hegemónico y las posiciones enunciativas}

El siguiente trabajo pretende abordar el discurso predominante o hegemónico sobre el DC/FM. Entendemos por discurso, aquellas prácticas verbalizadas y actuadas o acciones que producen y reproducen valores, ideologías y construcciones sociales, es decir, construcciones sobre los sujetos y los objetos a los que estas prácticas apuntan o señalan. Por tanto, un discurso equivale a una práctica (que puede ser verbal o bien, puede ser acción). Un discurso debe ser entendido como el uso institucionalizado del lenguaje y de sistemas simbólicos semejantes al mismo. Asimismo, los discursos pueden entrar en complicidades o competir unos con otros o bien crear versiones de realidades distintas e incompatibles. Por tanto, conocer algo significa conocerlo en términos de uno o más discursos (Iñiguez, 2003).

Como apuntan Davis y Harré (1990), el discurso es un proceso público polifacético a través del cual los significados se obtienen progresiva y dinámicamente. Las historias se localizan en varios discursos diferentes; de esta forma, varían notablemente en términos del lenguaje usado, los conceptos, los temas y los juicios morales relevantes y la posición del sujeto correspondiente. De esta manera, la experiencia de género, raza 
y clase que un sujeto incorpora a su identidad personal-social, puede sólo expresarse y entenderse a través de las categorías disponibles para ellas en el discurso.

El discurso psicológico o biomédico hegemónico en contextos de salud (consultas terapéuticas, jornadas de difusión, literatura científica, etc.) se caracteriza por una serie de elementos, conexiones y efectos que hemos analizado mediante una exhaustiva revisión de textos, manuales y publicaciones científicas, académicas, profesionales de la medicina o de la psicología. Los denominamos discurso hegemónico porque las prácticas y líneas de acción que sostienen son las que actualmente tienen más vigencia en la intervención del DC/FM. A la vez, hemos encontrado otros textos-discursos minoritarios más críticos que implican resistencias y conflictos, y a la vez posibilidades de cambio.

Utilizamos el concepto de "posición", "posición enunciativa" (2003) o "posicionamientos", precisamente porque da cuenta del lugar (o posición) desde donde enuncia y despliega el repertorio de acción cada discurso. Davis y Harré nos describen en qué consiste este posicionamiento del discurso, ligando las posiciones a agencias enunciantes que lo vehiculizan y lo ponen en práctica:

La fuerza constitutiva de cada práctica discursiva, creemos, se encuentra en la variedad de posiciones del sujeto. Una posición del sujeto incorpora un repertorio conceptual y la correspondiente ubicación en las estructuras de derechos para quienes usan ese repertorio. Una vez que se hace propia una posición particular, una persona inevitablemente percibe el mundo desde el punto de vista de esa posición privilegiada y en términos de imágenes particulares, metáforas, argumentos y conceptos relevantes dentro de la misma. La oportunidad nocional se encuentra inevitablemente incluida porque hay muchas y contradictorias prácticas discursivas en las cuales una persona podría participar. Incluso los participantes en este proceso son también producto de las prácticas discursivas (Davis y Harré, 1990: 244).

De esta forma, al hablar y actuar desde una posición, los agentes que la movilizan (sean instituciones o sean sujetos) producen unos efectos en ese contexto particular, efectos que tienen que ver con la constitución de los sujetos y objetos que entran en interacción, en nuestro caso, al hablar del discurso biopsicomédico, sobre las personas/enfermas y sobre el problema/enfermedad. Por tanto, si las posiciones disponibles en ese contexto particular marcan una dirección en la construcción de sujetos-objetos, también es cierto que se pueden negociar nuevas posiciones alternativas. Y es en este punto donde radica la capacidad analítica y política del uso de las "posiciones".

Se trata pues de identificar las posiciones de sujeto disponibles en esos discursos, y relacionarlas con las construcciones y acciones discursivas.

El sujeto asume el estatus de enunciador que define el discurso (o formación discursiva, según Foucault, 1970) en el que se encuentra. Pero hay que tener presente que un mismo sujeto puede activar distintas posiciones en un mismo discurso (es decir se sitúa 
en diferentes lugares de enunciación) (Iñiguez, 2003). Y también que distintos tipos de enunciados (variabilidad del discurso) pueden estar conectados a una misma posición o posicionamiento. Las instituciones delimitan el ejercicio de la función enunciativa, el estatus del enunciador y de los/as destinatarios/as, los tipos de contenidos que se pueden decir, las circunstancias de enunciación legítimas para tal posicionamiento, de ahí la conexión del discurso a las instituciones y al análisis del poder, del orden social, de la intersubjetividad y de procesos de transformación social. Para la obtención de "posiciones" hemos utilizado tres operaciones según Iñiguez (2003)³.

\section{Corpus textual para el análisis}

1.-El análisis realizado está basado en la recolección de material documental. Hemos seguido unos criterios teóricos muestrales consistentes en:

Considerar artículos científicos de carácter biomédico, psicológico y legales publicados en España. Concretamente: a) artículos de publicaciones científicas de carácter biomédico y psicológico; b) informes médico-psicológicos de asociaciones de fibromialgia; c) normativas legales y protocolos de actuación frente a la fibromialgia.

Estas fuentes constituyen la dimensión científica y objetiva del conocimiento, lo cual tiene un impacto sobre las intervenciones y prácticas cotidianas de los profesionales y los/as usuarios/as y familiares.

2.-Publicaciones en el intervalo temporal 2001-2013.

A partir de la búsqueda en la base de datos Dialnet ${ }^{4}$, seleccionamos 51 artículos y documentos (24 del ámbito biomédico; 20 del ámbito psicológico y 7 del ámbito nor-

3 a) la diferenciación texto-discurso, donde a partir de los relatos transcritos de los participantes o bien del material textual se obtienen los códigos, normas y sentido que llevan implícitos, lo cual nos permitirá la interpretación y ordenación de los datos;

b) la distinción locutor/a-enunciador/a, es decir "quien" vehiculiza ese discurso, desde que implícitos, intereses, afiliaciones y valores lo está poniendo en juego, lo cual también contribuye a la interpretación y ordenación posterior; y

c) la operacionalización del corpus, consistente en organizar, clasificar la información, introduciendo el concepto de "intertextualidad" que consiste en establecer la relación entre los discursos hallados en el texto.

${ }^{4}$ Portal de difusión de revistas científicas y humanísticas de España, Portugal y Latinoamérica, incluyendo también libros (monografías), tesis doctorales, jornadas y otro tipo de documentos. El portal se inicia en el año 2001. Encontramos 366 artículos (en 23 revistas científicas) y otros documentos (tesis, monografías y jornadas), a partir de la palabra de búsqueda "fibromialgia". Además de estos documentos, consideramos dos revistas con publicaciones frecuentes sobre fibromialgia procedentes de páginas web. (Sociedad Española del dolor crónico, INFOCOP- revista del Colegio de psicólogos-, y documentos de las páginas web de 4 Asociaciones españolas de fibromialgia. 
mativo-legal). Los criterios para elegir estos textos fueron tener presente la máxima variabilidad considerando el:

» Nivel de reconocimiento científico de la publicación: a) Revistas científicas y b) documentos de otro tipo de publicaciones (tesis, monografías, jornadas o congresos, guías, protocolos).

» Ámbito de intervención: a) Revistas médicas, b) revistas de enfermería y c) revistas de psicología.

» Perspectiva de la revista: a) feministas 5 , b) medico-farmacéuticas o c) de terapias alternativas-ocupacional-rehabilitación.

De manera que tras la lectura de los abstracts o resúmenes de cada artículo, consideramos que podíamos seleccionar una muestra de aproximadamente 51 artículos para representar la variabilidad de contenidos que se vehiculizan entorno a la FM desde las fuentes encontradas (ver anexo 1).

\section{Posiciones enunciativas del discurso biopsicomédico en torno al DC/FM}

Los resultados del siguiente trabajo muestran como el discurso biopsicomédico predominante en la ciencia y la intervención psicológica sobre DC/FM oscila entre tres posiciones diferenciadas y a la vez complementarias.

Hemos detectado una posición muy radicalizada y basada en parámetros biológicofisiológicos que centra su interés en la etiología de la enfermedad, su tratamiento y las consecuentes repercusiones psicológicas asociadas, mostradas a través de pruebas diagnósticas (lo que llamamos posición "cientifista"). La posición "cientifista" suele convivir con otra posición en la que el foco de interés es el mantenimiento de la relación ("adherencia terapéutica") entre personas afectadas-terapeutas (lo que llamamos posición "terapéutica de la conformidad"). Coexiste con ellas otra posición donde el discurso se centra en elementos relacionados con la gestión económica y legal de la enfermedad (costos directos e indirectos, absentismo laboral, invalideces...), así como en discriminar los casos "reales" de aquellos que no entrarían en el diagnóstico (posición "legal-economicista"). A continuación exponemos estas tres posiciones, y describimos para cada una de ellas a) cómo esta posición se caracteriza en los textos científicos; b) cómo construyen el problema o dolor crónico, y c) como construyen al sujeto/a que padece el problema:

${ }^{5}$ Las revistas que hemos seleccionado como feministas son aquellas que en su presentación lo hacen explícito. Las revistas feministas tienen un discurso crítico hacia la desconsideración social y familiar de la mujer que padece FM (Valls, 2008). 


\subsection{Posición "cientifista"}

Se constituye a partir de diferentes prácticas:

\subsubsection{Prioridad: diagnosticar el dolor}

En esta posición, el proceso de evaluación y diagnóstico del dolor crónico sin causa orgánica es fundamental. Utiliza autoinformes de síntomas, principalmente subjetivos, pero siempre acompañados por la presencia-evidencia de signos objetivos: "al menos once puntos localizados a lo largo del cuerpo, de respuesta al dolor". Estos puntos han sido anteriormente sistematizados y validados por científicos. La búsqueda de instrumentos de medida objetivos o de laboratorio que discriminen el cuadro de "fibromialgia" forma parte de los objetivos básicos. Tal como se observa en la siguiente cita;

El Colegio Americano de Reumatología (1990) señales tres criterios diagnósticos de la fibromialgia: historia de dolor difuso crónico de más de tres meses de duración, dolor en la presión en el menos once de los dieciocho puntos elegidos (nueve pares: occipucio, cervical bajo, trapecio, supraespinoso, segunda costilla, epicóndilo, glúteo, trocánter mayor y rodillas), y la ausencia de alteraciones radiológicas y analíticas (Wolfe et al., 1990:1).

Esta concepción mecanicista de la enfermedad, necesita de pruebas objetivas de daño en la estructura orgánico-corporal para afirmar o dar crédito del problema. Por lo tanto, la búsqueda de operadores que indiquen la intensidad de "síntomas fibromiálgicos" y la búsqueda de instrumentos de medida son vitales. Pero estos criterios provocan contradicciones cuando una mujer que acude por "fibromialgia" no cumple el cuadro de síntomas que figuran en el protocolo, entonces, el mecanismo de actuación es girar la responsabilidad hacia la propia mujer, con explicaciones como: "no discrimina bien", "exagera" o "tiene alguna dolencia hasta ahora desconocida":

Las Enfermedades orgánicas que provocan fatiga son las que más están cuestionadas y deslegitimizada por los profesionales sanitarios. (Valverde, 2010/11: 92).

La posición "cientifista" no considera ningún factor ligado a lo "no racional", como serían las emociones, la subjetividad, la experiencia particular. Todo lo contrario, descarta cualquier indicio de esta actividad, ya que como hemos comentado busca la objetividad y la racionalidad:

No hay evidencia respecto a que la FM sea una manifestación somática de alteraciones emocionales. Sólo un subgrupo de pacientes con FM, aproximadamente un tercio, presenta un nivel de síntomas psicopatológicos superior al hallado en otros trastornos de dolor reumático crónico de base orgánica (Lera, 2006: 41). 
A esta posición le interesa lo empírico, lo evidente y demostrable, por lo que solo logra detectar aquello que obedezca a esa regla, desconsiderando lo que no entre en esa lógica, sin cuestionarse que su metodología de detección sea reduccionista y excluyente (de lo subjetivo).

El uso de proliferación de tecnologías como los cuestionarios (ex. cuestionario de impacto de la "fibromialgia", escala de bienestar emocional del cuestionario de Salud y Calidad de Vida, etc...) así como las medidas lo más objetivables posibles del dolor son las tecnologías que legitiman la existencia de esta posición, así como también la existencia de la enfermedad (fibromialgia) a través de su confirmación por escalas medibles (Vololona, 2006).

\subsubsection{La causalidad psicosomática}

Acorde con el diagnóstico del dolor está la búsqueda de factores desencadenantes o productores de este y de la enfermedad. A pesar de que no hay un acuerdo unánime sobre la causalidad, sí que hay cierta coincidencia en afirmar que se trata de una multicausalidad de factores causales, sobre los cuales puede operar el discurso. A continuación introducimos un párrafo donde se hace referencia a esta idea:

Más bien, nos encontramos en un momento de la investigación de la fibromialgia en la que prevalecen los "quizás": quizás el problema que tienen estos pacientes sea una cuestión de recaptación de serotonina, quizás lo principal sea el anómalo funcionamiento del Sistema Simpático. (Ros, 2006: web).

También se mantiene una estrecha vinculación causal entre lo biofisiológico y las capacidades cognitivas, mentales, lo que provoca una autopotenciación de sus efectos. Como se enuncia en el siguiente párrafo:

Esto podría actuar directamente sobre el cerebro, explicando pues los hallazgos del funcionamiento cognitivo, o puede resultar en inflamación local, causando así los sintomas relacionados con la zona afectada del cerebro (Klimas, 2010: 3).

\subsubsection{Farmacologización}

El uso de fármacos (Carrasco et al., 2010) son el paradigma de tratamiento por el sufrimiento psicosomático y son las terapias que corresponden a esta posición. Hay un gran uso de los mismos, tal como se muestra en el párrafo.

Todos y todas las pacientes tomaban analgésicos y/o AINES, bien solos $(11,1 \%)$ o asociados a otros fármacos, principalmente antidepresivos (49,1\%) o relajantes musculares y/o ansiolíticos (24,6\%) (Ubago et al., 2005: 687). 


\subsubsection{Percepción de enfermo/a-síndrome}

La posición "cientifista" construye al sujeto/a como un enfermo/a caracterizado por un síndrome (conjunto de signos-síntomas) con difícil catalogación y diagnóstico médico. Estas dificultades provocan una falta de coordinación e interés por parte de los profesionales al no saber hacer frente al problema. Tal como se indica en el siguiente párrafo:

Un problema que nos encontramos es la falta de coordinación entre los especialistas y de unanimidad en el reconocimiento de la enfermedad como tal. Así, al no tratarse de una enfermedad "medible", "objetivable" y "replicable", su origen no filiado causa discrepancias. Actualmente, los neurólogos, en general, no están especialmente interesados en esta enfermedad; los psiquiatras, tienen saturación de casos ante la gran derivación de estos pacientes por parte de otros especialistas para ser tratados por la polémica depresión; los médicos internistas tampoco la encuadran habitualmente; e incluso, no todos los reumatólogos creen en la existencia de esta enfermedad (Ros, 2006: web).

El/la paciente acaba siendo un engorro para el ejercicio profesional, al no poder ser catalogado con facilidad como "enfermo de", situándose en un terreno ambiguo al definirse como un síndrome con deficiencias orgánicas, neurológicas, junto con una función cognitiva deteriorada. Tal como se apunta en el fragmento:

Aunque estos datos muestran una evidencia clara de que los pacientes con síndrome de fibromialgia tienen una función cognitiva deteriorada, debido al elevado número de factores que influyen en el rendimiento cognitivo que no fueron valorados en este estudio y que parte del rendimiento cognitivo no fue explicado por el nivel de dolor y ansiedad, no deben descartarse otras hipótesis relacionadas con la coexistencia de alteraciones en el sustrato neurológico central junto a deficiencias asociadas a estas u otras variables clínicas sintomáticas. Por estos motivos, los autores consideran necesarias futuras investigaciones que determinen las causas del deterioro neuropsicológico en este grupo de enfermos (Munguía-Izquierdo et al., 2008: 431).

A pesar de que en esta posición se barajan datos estadísticos sobre la cantidad de mujeres que padecen el síndrome, no se valora la organización social que hace del genero una variable relevante en el desarrollo y mantenimiento de la enfermedad, en su comprensión, sus efectos y tratamiento, o como diría Valls-Llovet (2010), no se realiza sistemáticamente un diagnóstico diferencial del dolor en función del sexo-género. El sexo-género sólo es una variable descriptiva y orientativa usada desde la epidemiología para focalizar más intensamente la mirada y las correlaciones de otras variables con el sexo-género o colectivo mujer que aqueja dolor, pero no una variable analítica y que posibilita un tratamiento específico. 


\subsection{Posición "terapéutica de la conformidad"}

\subsubsection{Prioridad: adherencia terapéutica}

Esta posición focaliza la importancia de la relación terapéutica entre profesionales y persona/s que sufre/n el dolor crónico. Por un lado en cuanto al acompañamiento y seguimiento del / la paciente, por otro en cuanto a la adherencia terapéutica.

Se apoya en la posición "cientifista" para justificar el acompañamiento y tratamiento psicológico, relacional y de adhesión terapéutica y con ello consigue autopotenciar su función. Tal como muestra el siguiente párrafo:

El dolor crónico es, principalmente, el resultado de un fallo en los sistemas naturales de analgesia ligados, generalmente, a una condición sensorial concreta y adicionalmente, y a consecuencia de ello, a la falta de recursos personales (psicosociales) para afrontar las implicaciones vitales que ello supone. (Vallejo: 2010: web).

Así, cuando se han agotado las alternativas médicas, es cuando aparece "oficialmente" la figura del psicólogo/a, para ayudarlos a cambiar actitudes y sobrellevar el dolor, enseñándoles a relajarse y a reconocer sus límites (Ros, 2006: web).

A partir de este presupuesto propugna la necesidad de asistir a consultas sucesivas y de seguimiento con especialistas (Martínez y Paterna, 2003). Con el concepto de cronicidad alcanza relevancia la adherencia y el vínculo terapéutico, con el consiguiente control que supone (del sistema sobre los/as usuarios/as):

Para que ello sea posible, será preciso elaborar previamente una cuidadosa historia clínica donde se detecten todos los posibles estresores actuales y pasados, $y$ donde quede reflejada la personalidad del paciente. En presencia de estos datos se verá qué se puede hacer para eliminar o modificar estos estresores, modificar la escala de valores, posible psicoterapia, etc. (Sedó y Ancochea, 2002: 563).

No obstante, se establece una crítica centrada en el paciente cuando la enfermedad no remite: se le hace responsable de que "no sigue el tratamiento adecuadamente" o bien, "no acepta o aprende a vivir con el dolor":

Se suele argumentar, como una forma de evitar el problema, que el dolor es subjetivo y que, por tanto, hay que acostumbrarse a vivir con él, arguyéndose en algunos casos que "de fibromialgia no se muere, ni se degenera" (Ros, 2006: web).

\subsubsection{Importancia de la terapia psicológica}

Desde la perspectiva psicológica, el tratamiento alcanza sentido desde el uso principalmente de terapias psicológicas denominadas cognitivo-conductuales, desde marcos 
de cognición que utilizan las / los pacientes para organizar la experiencia de vida y las prácticas cotidianas. Tal como se muestran en algunas citas:

...Actuar sobre los aspectos comportamentales y emocionales relacionados con el afrontamiento del dolor, mediante el abordaje tradicional de la terapia cognitivo-conductual con las aportaciones recientes de la terapia de aceptación y compromiso y otras terapias de conducta de tercera generación (Vallejo, 2010: web).

Estos tratamientos son criticados por algunos profesionales implicados en el dolor crónico sin causa orgánica. Podemos ver la siguiente declaración de una profesional del mundo de la salud afectada por FM que contrasta con el discurso anterior:

Otras psicoterapias brillan por su ausencia en estas enfermedades en las que los afectados tanto apoyo necesitan para poder recuperar su dignidad... Cuando una enfermedad causa cambios tan radicales como lo hacen la FM, y cuando existe tal marginalización social, una terapia que consiste en cambiar pensamientos "negativos" por "positivos" con la ayuda de una goma de pollo, evitando ir a un nivel más profundo, difícilmente puede ser de utilidad. También el que al paciente se le esté repitiendo que sus pensamientos son "inadaptados" es, sencillamente, una agresión que va contra el juramento hipocrático de "primero, no hacer daño" (Valverde y Markez, 2009: 89).

La posición "terapéutica de la conformidad" también se hace extensiva a otros entornos de relación del/la paciente, se trata de los espacios asociativos, donde la "terapia relacional" se constituye como el recurso principal al DC del colectivo de pacientes:

Actualmente, son las asociaciones civiles, como por ejemplo, la Asociación Valenciana de Afectados de Fibromialgia (AVAFI) y los psicólogos desde la práctica clínica privada quienes están ofreciendo una mayor asistencia psicológica a los afectados de esta enfermedad; a pesar de que son los propios médicos los que aconsejan a los afectados de fibromialgia que recurran al apoyo psicológico (Ros, 2006: web).

\subsubsection{Sujeción del/la paciente}

Esta posición apunta a un/a sujeto/a con rasgos prototípicos de personalidad o un perfil "psicológico" específico. Tal como muestra el siguiente párrafo, coincidiendo con otras descripciones similares encontradas en varios artículos:

Suelen ser personas activas, perfeccionistas, impacientes, con mucha capacidad para asumir responsabilidades ajenas, dependientes emocionalmente, nerviosos, con dificultad para poner límites, con un "locus de control" externo, etc. (Ros, 2006: web). 
Sorprende que en ningún momento, la condición de "ser mujer" esté explícita en el discurso de la relación terapéutica, a pesar de que en lo implícito se conecta mujer con DC/FM. En los párrafos encontrados se destaca el uso en el lenguaje del uso masculino (por ejemplo, los afectados, enfermos, nerviosos). En esta posición, también se desconsidera el carácter sexuado del DC/FM (especialmente padecida por mujeres), y en su defecto, se considera un referente masculino para pensar en la intervención. Según Sara Velasco (Velasco, 2009) es el modelo de salud carente de considerar los atributos sociales, las normas y roles de género, los ideales y componentes subjetivos de identidades femeninas y masculinas.

Desde esta posición también se observa cómo se concibe la existencia de una persona sumisa al tratamiento, dependiente del terapeuta, pero a la vez con requerimientos de ser responsable, activa e implicada en la autoadministración de las pautas terapéuticas para ser reconocida como enferma y obtener expectativas de resolución a sus problemas. En el siguiente párrafo encontramos un protocolo de las diferentes etapas de tratamiento psicológico por las que pasa un/a paciente hasta llegar a su mejoría. En estas etapas se refleja la exigencia que se cierne sobre el sujeto desde una posición "terapéutica de la conformidad"( "de dominio y control personal", de autogestión, —en el segundo párrafo-):

El PSOCQ incluye cuatro escalas (Precontemplación, Contemplación, Acción, y Mantenimiento) que describen el grado en que se progresa o retrocede en la preparación para adoptar y aceptar la autogestión. Se ha proporcionado evidencia sustancial de la fiabilidad y validez sobre los cuatro estadios. En el estadio de precontemplación, no parece existir intención de cambiar ("todo esto sobre cómo afrontarlo mejor es una pérdida de tiempo"), en la contemplación se constata ya una toma de conciencia del problema del paciente y una intención de cambiar en breve ("he asumido recientemente que depende de mi enfrentarme mejor a mi dolor"), en la acción se llevan a cabo cambios manifiestos de comportamiento ("estoy probando algunas habilidades de afrontamiento para manejar mejor mi dolor), $\boldsymbol{y}$ en el mantenimiento se tratan de conservar estos cambios ("he incorporado a mi vida cotidiana técnicas para sobrellevar mi dolor") (Merayo et al., 2008: 336).

Probablemente estas personas mantuvieron creencias acerca de que el dolor es un problema de naturaleza estrictamente médico-orgánico y que, por tanto, no requería la adquisición de habilidades de autogestión.... los tratamientos psicológicos como los realizados en nuestro estudio pueden promover mejores resultados, no sólo desarrollando nuevas habilidades, sino también generando expectativas de control y autoeficacia sobre el problema a través de experiencias de dominio y control personal durante el programa de cambio. (Merayo et al., 2008: 337).

Así pues, se visualiza que esta posición "psicologiza" a las personas afectadas (mujeres principalmente), ante la dificultad en la capacidad explicativa de este dolor crónico sin 
causa orgánica que manifiestan. Estos procedimientos dificultan la exploración de otras hipótesis diagnosticadas y deslegitiman la presencia de ciertos síntomas. Los hombres (de carne y hueso) tampoco aparecen en el marco de la enfermedad (aunque se utiliza un lenguaje con referente masculino), y cuando alguno manifiesta síntomas, aparecen como excepción.

\subsection{Posición "legal-economicista"}

Esta posición es más minoritaria, pero también la encontramos en una parte del discurso biopsicomédico, concretamente más ligada con la posición "cientifista" — en cuanto busca la objetividad y la evidencia de los hechos-. Está conectada por un lado, al discurso legal, en tanto que debe estar sustentada por normas y leyes que promuevan derechos y deberes de las personas que padecen el problema-enfermedad. Por otro lado, al discurso económico, en tanto que regula los gastos económicos que supone la enfermedad-problema, pero a la vez, se usa como generadora de recursos y productora de servicios.

\subsubsection{Prioridad: legitimar la enfermedad}

Esta posición permite afianzar la legitimidad alcanzada con el diagnóstico de la posición "cientifista" mediante dos elementos claves: la peritación (como instrumento objetivo de medida que garantiza el grado de incapacidad) y la credibilidad del/la afectado/a. Desde una "posición legal-economicista", la enfermedad también se constituye como una desconocida, frente a la imprecisión o inexistencia de diagnóstico, reclamando velar por la instauración de pruebas y evidencias objetivas que puedan "medir" el dolor, la incapacidad para que el sistema legal y socio-laboral pueda compensar a las personas afectadas, o bien detectar o discriminar a aquellas personas que intentan engañar al sistema. Tal como se comenta en el siguiente párrafo:

Al desconocerse la etiología de la enfermedad y su tratamiento, nos encontramos, por una parte, con muchas pacientes sin diagnosticar; y por la otra, con muchos diagnósticos realizados sin mucha precisión y que no han sido revisados con posterioridad (Ros, 2006: web).

Esta posición se convierte en garante de la verdad (credibilidad de lo que testifican las personas afectadas). En este sentido, el discurso introduce todo aquello que tenga que ver con la peritación, con el desarrollo de instrumentos y protocolos de evaluación específicos, y con la detección y medición del dolor crónico para poder dar respuestas:

$Y$ es que, para la declaración de incapacidad permanente, se exige, de conformidad con el artículo 136 de la Ley General de la Seguridad Social, que a las 
dolencias sean "susceptibles de determinación objetiva", lo que obliga, tanto a los efectos de acreditar la existencia de una incapacidad permanente como a los efectos de calificar el grado de incapacidad permanente, a una objetividad no siempre fácil de concretar en una enfermedad cuyo diagnóstico es exclusivamente clínico, como es la fibromialgia (Lousada, 2009: web).

Se señala una constante misión hacia la detección del fraude, de la simulación de la enfermedad. Hemos encontrado algunos textos donde se resalta esta idea, la cual, construye un imaginario de paciente "potencialmente simulador":

..es evidente que la colaboración entre el conocimiento científico de la Psicología y la Medicina es fundamental para implementar estrategias para detección eficaz de la simulación del dolor y la discapacidad. En el contexto clínico médicolegal, las patologías más simuladas suelen ser aquellas cuya sintomatología resulta difícil de relacionar claramente con una base orgánica objetivable (Gonzalez y Capilla, 2009: web).

La misión de esta posición se reviste de autoridad (acompañada por la "cientifista" y la "terapéutica de la conformidad") al decirnos el "verdadero estatus del paciente", reconociendo sus derechos simbólicos y materiales, es decir, la posibilidad de ser atendido y reconocido legal y económicamente frente a la sociedad. Ello se hace evidente cuando las/los pacientes no son diagnosticados, peritados, reconocidos:

Por inaudito que pueda parecer, estos enfermos peregrinan durante años de una a otra consulta, bajo la sospecha velada (y no tan implícita en muchos casos) acerca de la veracidad de su dolencia; confundiéndose, a veces, con "un acto de fe". Suelen acabar "aparcados en el médico de cabecera", y experimentando tratamientos paramédicos (Ros, 2006: web).

Por tanto, esta posición está muy en coherencia y se retroalimenta con elementos que propugnan las otras dos posiciones, y en tanto que se postula desde un conocimiento "aséptico", "racional" "positivista" y "descontextualizado", podemos decir que proviene de una ciencia androcéntrica (Haraway, 2004), la cual desconsidera los valores subjetivos, locales y situados, donde no existe "el otro particular" con sus características específicas, sino un "otro generalizado" que desconsidera las condiciones de producción donde se produce su experiencia, en nuestro caso, su experiencia de dolor (Pujal et al., 2009).

\subsubsection{Tratamiento: sobre la incapacitación de la persona}

En la posición "economicista-legal" la persona es construida como incapacitada, como alguien que no es productiva para el sistema, sino que requiere de sus recursos. Previo al diagnóstico, puede ser percibida como potencialmente simuladora de una enferme- 
dad, o bien exagerada en sus síntomas con la idea de conseguir recursos económicos y de atención psicosocial:

La simulación, es decir la producción intencional de síntomas físicos y/o psicológicos falsos o exagerados motivados por incentivos externos, es un problema con el que se encuentran frecuentemente los profesionales de la salud en su práctica clínica, en especial en aquellos ámbitos donde el paciente puede percibir prestaciones económicas de algún tipo y la asistencia es gratuita. Los datos indican que en este contexto las conductas de simulación más frecuentes son la exageración de síntomas (dolor) y exageración de la discapacidad, por la posibilidad de obtener incentivos económicos; mientras que, la simulación de sintomatología psicopatológica sería más prevalente en contextos forense - criminales, con el fin de reducir / evitar el pago de indemnizaciones, condenas y encarcelamientos (González y Capilla, 2009: web).

El colectivo de enfermos/as se percibe como un colectivo real y potencialmente a tener en consideración - tanto por su cantidad, como por su grado de incapacidad - como receptor de ayudas por parte de un sistema que debe ofrecerle considerables recursos:

El $20 \%$ de las personas con la fibromialgia, hoy en día, están invalidadas por su enfermedad para trabajar. En España, según un estudio de la Sociedad Española de Reumatología, la FM es la enfermedad músculo-esquelética que más bajas produce por incapacidad laboral (EPISER 2000). Aun así, sólo un pequeño porcentaje reciben prestaciones (Valverde, 2000: 28).

De ello se deriva la percepción de personas que padecen DC/FM como una carga por las prestaciones sociales y económicas que requieren, por tanto, como un colectivo consumidor de recursos públicos. También en esta posición, se marca la diferencia mujer-hombre en la construcción del estereotipo de enfermo/a ya que lo relaciona con atributos sociohistóricamente asignados a la categoría mujer, tal como: "dependiente", "demandante" "exagerada" "falta de discriminación", "mentirosa", "no controladora", etc., lo que atribuye una feminización al/la paciente, y una consecuente patologización femenina. Este hecho puede resultar notorio de cara al diagnóstico o peritación de fibromialgia en hombres, debido a que al identificarla con atributos de estereotipo femenino, cuesta más reconocer su padecimiento en el sexo-género masculino (tanto para profesionales y peritos, como para los propios hombres que puedan padecerla).

\section{Conclusión}

A partir del análisis discursivo de artículos científicos sobre fibromialgia, hemos encontrado tres posiciones que constituyen lo que hemos denominado el discurso biopsico- 
médico. Estas posiciones se potencian mutuamente: a) la posición "cientifista" que se centra fundamentalmente en procesos discriminatorios del diagnóstico y en la farmacologización del tratamiento de una enfermedad considerada orgánica, obviando elementos humanizadores; b) la posición "terapéutica de la conformidad" que implica la sumisión al modelo biopsicomédico, especialmente al seguimiento de las pautas de tratamiento psicológico cognitivo-conductual y al reclamo de que las personas afectadas, especialmente las mujeres, deben encajar en unos "moldes" para ser reconocidas como enfermas y una adherencia a la terapia y al terapeuta; y c) la posición "económico-legalista" que se sustenta en los instrumentos diagnósticos (test, anamnesis..) de las otras posiciones para asegurar la credibilidad del paciente y la veracidad de su enfermedad, y a partir de ahí, justificar sus intervenciones a través de facilitar recursos materiales o adquisición de derechos a través de normativas aplicables a las pacientes de DC/FM. Cada una de estas posiciones se muestra conformando una definición de problema/enfermedad y una identidad de sujeto/paciente como hemos visto en el análisis.

Como ya comentamos al inicio de este trabajo, no se ha contemplado los discursos provenientes de los propios profesionales que hacen intervención con pacientes, o la de los familiares o la de las propias pacientes que experimentan DC/FM con el fin de recoger otras posiciones, sin embargo, el conocimiento de este contexto a través de los análisis efectuados en otros trabajos en base a entrevistas a pacientes, familiares y terapeutas (ver Pujal et al., 2011), nos ha facilitado la interpretación. Es importante ampliar el campo de mirada para ver otras alternativas e integrar otras perspectivas desde una variabilidad de posiciones para comprender a fondo el fenómeno.

Una lectura desde una perspectiva crítica y de género sobre estas posiciones que comporta el discurso biopsicomédico, nos indica algunos puntos a tener en cuenta en futuras intervenciones sobre la FM/DC:

\subsection{Explicaciones individualistas y agenéricas carentes de contexto social}

En los resultados de las tres posiciones detectadas, el discurso biopsicomédico se representa como un modelo autoritario y de enfoque individualista, que no considera la perspectiva de género en sus posiciones, es decir, incorporar qué factores psicosociales en la experiencia de las mujeres han podido tener relación con la aparición del DC/FM (Le Breton, 1999). Este discurso biopsicomédico se identifica con lo racional, objetivo y la verdad, descartando los elementos "no racionales" que configuran el malestar de las personas, mujeres, afectadas. Excluye la intervención sobre el orden social donde se enmarca razones estructurales y de sexo-género de donde emerge el problema, razones cómo serían las historias de vida de mujeres que reflejan la gran sobrecarga que han llevado a través de los cuidados que han dispensado a otros.

La enfermedad alcanza un lugar central en la vida de las mujeres, de manera que se trata de "un vivir en la enfermedad" más que de "un vivir con la enfermedad". Tal es 
así, que la persona debe regular su capacidad energética, los recursos para hacer más o menos cosas, autocontrolarse, planificar lo que puede hacer o no, llevar una disciplina de su cuerpo, de sus movimientos, de su ritmo de vida, de su tiempo. En este sentido la enfermedad constituye una tecnología de control sobre la vida y el cuerpo de la persona, de las mujeres. Ello se constata en las posiciones encontradas en el análisis en tanto que esos discursos propugnan prácticas reguladoras y de sujeción a lo patológico, en relación al dolor cronificado que precisa medicación, afectaciones neurológicas, alteraciones cognitivas, autocontrol y autogestión de la persona, adherencia terapéutica a las pautas, necesidad de "decir la verdad" o ser creíble.

\subsection{Sujeción de las mujeres como condición de su existencia}

En general, la construcción de identidad de enfermo/a sirve a la persona simbólica y materialmente. Simbólicamente, porque le permite tener presencia, representación social, institucional y familiar; materialmente, porque le reporta ganancias y pérdidas en su estatus de vida, afectos y roles que desempeñaba hasta el momento. Muchas mujeres, tras saber su diagnóstico dicen: «estoy mejor una vez que sé que no me lo estoy inventado». Ya hemos visto las dificultades de credibilidad por parte del contexto que rodea a la persona que sufre dolor crónico sin causa orgánica (Valverde et al., 2009). El diagnóstico "fibromialgia" conlleva nombrar lo que "no se puede llamar". Es decir, que alguien pueda nombrar lo que las personas afectadas no pueden nombrar, lo que permite reconocimiento desde una institución poderosa, una institución de poder que dice la "verdad". La intervención sobre este dolor, legitima y regula el desarrollo de las prácticas y el diagnóstico médico-psicológico, actúa como un simbólico en el sentido de poner límites a su posibilidad de extensión.

Pero también debemos analizar, desde las relaciones de poder social, que implica a cambio esta transferencia de posibilidades para el sujeto. En el caso que hemos analizado, vemos que hay una "sujeción" a la normativa y al discurso biopsicomédico, vemos que "no hay ganancia de voz" por parte de las mujeres o las personas que la padecen, porque no hablan ellas, habla el sistema a través de ellas (tecnología del poder) (Pujal y Mora, 2009). Esta sujeción es velada, no es nada fácil darse cuenta o resistirse a ella. Tal como diría Butler (2001): el sujeto debe ser sujetado en su categoría de "fibromialgica" para existir.

\subsection{Resignificar-repolitizar el dolor}

Llegado a este punto, nos queda pues recoger aquellos elementos emergentes de la crítica anterior para elaborar una propuesta. Esta propuesta es el intento de transformar la fenomenología y las bases epistémicas de las posiciones estrictamente biopsicomédicas 
y abrir nuevas vías de comprensión-intervención bio-psico-socio-histórica sobre el problema del DC/FM, de manera que deje de ser un enigma, o una "caja negra". Entender el problema desde los condicionantes sociales que sitúan a las mujeres en una posición vulnerable, como muestra el trabajo de Pujal-Llombart y Mora (2013) cuya propuesta es llegar a un "diagnóstico psicosocial de género" que recoja la historia de vida olvidada de las mujeres (Pujal et al., 2011), rompiendo el reduccionismo de la construcción de la enfermedad que el sistema psicomédico promueve, especialmente porque no escucha a las/los pacientes. Esta propuesta es fundamental para volver a resignificar el DC/FM, a partir de la cual debe constituirse una nueva política en la que el dolor sea tomado como una tecnología de poder social, de poder institucional. Una política en la que se desvele y se subvierta la disciplina que se infringe en los cuerpos de las mujeres y donde se cuestione la autoridad de las instituciones que se autoatribuyen toda la capacidad de resolver el problema. También, como propone Correa-Urquiza (2015) se trata de un acto de "desenfermar", o de permitir una identidad a las personas afectadas que esté fuera de la noción de dificultad, de incapacidad, que habilite otro tipo de posibilidades.

\section{Bibliografía}

ACAF. Asociació Catalana d'Afectats de Fibromialgia (2005): Presentació de l'asociació. Comunicació presentada a Taula rodona, Barcelona. Collegi Oficial de Psicòlegs de Catalunya.

BARKER, K. (2005): The Fibromyalgia Story. Medical authority and women worlds of pain, Philadelphia, Temple University Press.

Benhabib, S. y D. Cornell (1987): Feminism as Critique: Essays on the Politics of Gender in Late-Capitalist Society, Cambridge, Polity Press.

Burin, M. (1990): El malestar de las mujeres. La tranquilidad recetada, Buenos Aires, Paidós.

BUtLER, J. (2001): Mecanismos psíquicos del poder: teorías sobre la sujeción, Valencia, Feminismos, Universidad de Valencia.

Carrasco, M.C., C. Jiménez y M. MÁrquez (2010): "Fibromialgia: cuando el dolor es una historia de vida", Index Enfermeria, 19, pp. 2-3.

Conrad, P. y J. W. Schneider (1992): Deviance and medicalizations. From badness to sickness, Philadelphia, Temple University press.

Correa-Urquiza, M. (2015): Radio Nikosia. La rebelión de los saberes profanos, Madrid, Grupo5.

DAVIES, B. y R. HARRÉ (2009): "Positioning: the discursive production of selves", Journal or the Theory of social Behaviour, 1990, 20, pp. 43-63. 
(2007): "Posicionamiento: La producción discursiva de la identidad", Athenea Digital. Disponible en:

$<$ http://psicologiasocial.uab.es/athenea/index.php/atheneaDigital/article/ view/445/362HYPERLINK > [Consultado el 20 de octubre de 2012].

Delvecchio M-J, P.E. Brodwin, B.J. Good y A. Kleinman (eds.) (1992): Pain as human experience: An anthropological perspective, California, University of California press.

Foucault, M. (1970): El orden del discurso, Barcelona, Tusquets.

Foucault M. (1980): El nacimiento de la clínica: una arqueología de la mirada médica, Editorial Siglo XXI, México.

González, H. y P. Capilla (2009): Detección de la simulación del dolor crónico. Un enfoque multidisciplinar. Disponible en:

$<$ http://www.infocop.es/view_article.asp?id=2380\&cat=38)> [Consulta 15 enero de 2013].

Haraway, D. (2004): Testigo_Modesto@Segundo_Milenio: HombreHembra@_Conoce_ Oncoratón ${ }^{\circledR}$. Feminismo y tecnociencia, Barcelona, Editorial UOC.

Illich I. (1975): Némesis médica: la expropiación de la salud, Barcelona, Barral Editores.

IÑIguez, L. (2003): El análisis del discurso. Manual para las Ciencias Sociales, Barcelona, Editorial EDIUOC.

Klimas N. (2013): Sobre Fibromialgia, V.I.H. y Síndrome de Fatiga Crónica. Plataforma Nacional para FM, SFC, SQM. Reivindicación de derechos. Disponible en:

$<$ www.plataformafibromialga.org ${ }^{a}$ [Consultado el 20 de enero de 2013].

Le Breton, D. (1999): Antropología del dolor, Barcelona, Seix Barral, Los Tres mundos.

LERA, S. (2006): Características y respuesta al tratamiento multidisciplinar de pacientes afectadas de fibromialgia, Tesis doctoral inédita, Universitat de Barcelona, Departament de Psiquiatria i Psicobiologia clínica, Programa de doctorat: 'Recerca i clínica dels trastorns mentals'. Bienni 1998-2000.

LuZ, M.T. (2011): "El desafío de la salud en las ciencias sociales: el caso de Brasil”, Política y Sociedad, 48 (2), pp. 313-327.

LousADA, J. F. (2009): El tratamiento jurídico de la fibromialgia en perspectiva de género. Disponible en:

$<$ http://www.afibrosal.org/documentos/legislacion/juridicojuridicoFibr.pdf $>$ [Consultado el 15 de enero de 2013].

Martínez, M. C. y C. Paterna (2003): “Comprender la Fibromialgia desde una perspectiva psicosocial femenina”. Comunicación presentada en el Congreso Nacional de Psicología Social, Málaga, España.

Merayo, L.A., F.J. Cano y L. Rodríguez (2008): "Motivación para el cambio como predictor de la adherencia terapéutica en el dolor crónico", Apuntes de Psicología, 26 (2), pp 331-339. 
Munguia-Izquierdo, D., A. Legaz-Arrese, D. Moliner-Urdiales y J. Reverter-Masia (2008): "Neuropsicología de los pacientes con síndrome de fibromialgia", Psicothema (20) 3, pp. 427-431.

Pastor, M., A. Lledó, M. Martín-Aragón, N. Pons, J. Rodriguez-Marín, S. Sánchez, M. Terol, J. Fernández, J. Tovar, I. Ibero, J. Rosas y E. Salas (2003): “Afrontamiento y estado de salud en la Fibromialgia", Comunicación presentada en el Congreso Nacional de Psicología Social, Oviedo, España.

Pujal, M. y E. Mora (2009): "Aproximación al cuerpo fibromiálgico como respuesta ambivalente a una poli-violencia de género", en Actas del I Congreso Internacional sobre género: la cultura en el cuerpo, Elche, SIEG, 11-13 noviembre 2009.

Pujal, M., E. Mora, P. Albertín, B. Mestres y P. Amigot (2011): "Las voces silenciadas en tiempos de "igualdad". El dolor desde una perspectiva de género", Memoria de investigación presentada al Institut Català de les Dones, Catalunya.

Pujal i Llombart, M. y E. Mora (2013): "Trabajo, dolor y su diagnóstico psicosocial de género. Un ejemplo", Revista Universitas Psicológica, 12 (4), pp. 1181-1193.

Rivera, J. (2009): “Tratamiento farmacológico en fibromialgia", V Jornadas Codo con Codo, Fundación FF Madrid.

Rodríguez, R.M. (1999): Foucault y la genealogía de los sexos, Barcelona, Anthropos Editorial.

Ros, N. (2006): Intervención psicológica en la fibromialgia. Disponible en:

$<$ http://www.infocop.es/view_article.asp?id=987> [Consultado el 15 de enero de 2013]

Ruiz I., M.C. Ubago, MJ. Bermejo, J. Plazaola, A. Olry, E. Hernández (2007): “Diferencias en características sociodemográficas, clínicas y psicológicas entre hombres y mujeres diagnosticados con fibromialgia”, Revista Clinica Española., 207 (9), pp. 433-439.

Sedó, R. y J. Ancochea (2002): "Una reflexión sobre la fibromialgia”, Atención Primaria, 29 (9), pp. 562-564.

Ubago, M.C., I. Ruiz, M.J. Bermejo, A. Olry y J. Plaza (2005): “Características clínicas y psicosociales de personas con fibromialgia. Repercusión del diagnóstico sobre sus actividades", Revista Española de Salud Pública, 79, pp. 683-695.

Valverde, M. (2000): "Prevalencia de la fibromialgia en la población española. Estudio EPISER", Revista Española Reumatología, 27, p. 157.

Valverde, M., J.A., Rivas y L. Carmona (2001): Estudio EPISER. Prevalencia e impacto de las enfermedades reumáticas en la población adulta española, Madrid, Merck Sharp \& Dohme (MSD) y Sociedad Española de Reumatología, pp. 77-91.

Valverde, C. e I. Markez (2009): "Lo que tú tienes que hacer es...: El uso coercitivo de Terapia Cognitivo - Conductual en las nuevas enfermedades", Norte de Salud Mental, 35, pp. 89-106. 
Valverde, C., I. Markez y C. Visiers (2009): "Breves intervenciones en el largo viaje: la comunicación con pacientes con fibromialgia o síndrome de fatiga crónica", OMeditorial, Colección Prometeo.

VALVERDE, C. (2010-2011): “'Los cuerpos del delito': injusticias y oportunidades en los Síndromes de Sensibilidad Central", Papeles de relaciones ecosociales y cambio global, 112, pp. 141-153. Disponible en:

$<$ http://www.ligasfc.org/wp-content/uploads/2011/02/los-cuerpos-del-delito_C.VALVERDE.pdf $>$ [Consultado el 20 de marzo de 2013].

Valls, C. (2008): "Diagnóstico diferencial del dolor y la Fibromialgia", Anuario de Psicología, 39 (1), pp. 87-92.

Valls-Llovet, C. (2010): "La salud bio-psico-social regenerada". Monográfico Desigualdades de género en "tiempos de igualdad". Aproximaciones desde dentro y fuera de la/s psicología/s, Quaderns de Psicologia. International Journal of Psychology, 12 (2), pp. 175-181.

VAlLejo, M.A. (2010): La terapia cognitivo-conductual es el tratamiento psicológico que más evidencia aporta sobre el dolor crónico. Disponible en:

$<$ http://www.infocop.es/view_article.asp?id=2784> [Consultado el 2 febrero de 2013]

Velasco, S. (2009): Sexos, género y salud, Madrid, Minerva Ediciones.

Vololona, R. (2006): "From representation to mediation: The happing of collective mobilization on muscular dystrophy in France", Social Science \& Medicine, 62, pp. 564-576.

Wolfe F. et al. (1990): "The American College of Rheumatology 1990 Criteria for the Classification of Fibromyalgia", Report of the Multicenter Criteria Committee, Feb 33 (2), pp.160-72.

\section{Anexos}

TeXTOS PARA EL ANÁLISIS DEL DISCURSO BIOPSICOMÉDICO

\section{1. Ámbito de la Bio-medicina}

Anciones , B.; Gómez-Argüelles, J.M.; Prevalencia de síntomas neurológicos asociados a la fibromialgia. Rev Soc Esp Dolor 16 (2009);4 :222 - 229.

Azkue, J.J.; Bilbao , J.; Montoya, P.; Aira, Z.; Buesa, I.; Salgueiro, M.; :Valoración de factores sociales y clínicos en el síndrome de fibromialgia. Rev Soc Esp Dolor 16 (2009);6:323-329

Biorritmes. Revista de Fibromialgia y Síndrome de Fatiga Crónica.

$<\mathrm{http}: / /$ www.biorritmes.com/> 
Carrasco Acosta, M.C.; Jiménez de Madariaga, C.; Márquez Garrido, M. Fibromialgia: cuando el dolor es una historia de vida. Index Enferm v.19 n.2-3 Granada abr.-set. 2010

Castel-Bernala, V. de Felipe García-Bardóna y J. Tornero-Molina. Evaluación psicológica en el dolor crónico. Reumatol Clin. 2006; 2 Supl 1: S44-9

Collado et al.; Protocolo de evaluación y tratamiento de los pacientes con fibromialgia. Unidad de Fibromialgia Instituto de Especialidades Médico-Quirúrgicas Instituto de Neurociencias Anestesia-Clínica del Dolor. Hospital Clínic. Corporación Sanitaria.

DiARIO MÉDICO INTERACTIVO. La fibromialgia provoca un gasto de 10.000 euros por paciente al año. Rev Soc Esp Dolor 16 (2009); $7: 417$ - 418.

$<$ http://revista.sedolor.es/articulo.php?ID $=647>$

Deus, J.; ¿Se puede ver el dolor?. Reumatol Clin.2009; 5(05) :228-32.

Echevarría Moreno, M.; Herrera Silva, J.; Morales Muñoz, C.; Bullón Barrera, F.; Fernández Muñoz, I. ; Mayorga Buiza, M.J.: Impacto de un programa de educación sanitaria en pacientes con fibromialgia. Rev Soc Esp Dolor 17 (2010);5 :227 - 232

Echevarría, M.; Caba, F.; Mayorga, M.J.; Villegas, A.; Morales, C.; :La población fíbromiálgica ante la cirugía. Estudio descriptivo y análisis del dolor posoperatorio en histerectomías. Rev Soc Esp Dolor 17 (2010);5 :233 - 238

EsCUDERo-CARRETERo, M. et al. Fibromialgia: percepción de pacientes sobre su enfermedad y el sistema de salud. Estudio de investigación cualitativa. Reumatol Clin.2009. $<$ http://www.elsevier.es/sites/default/files/elsevier/eop/S1699-258X(09)00157-0.pdf>

García-Fructuoso, F. Síndrome del dolor neuroplasticamente inducido. Servicio de reumatología Clínica CIMA. Barcelona.

Kuimas N.; Sobre Fibromialgia, V.I.H. y Síndrome de Fatiga Crónica. (2010). Plataforma Nacional para FM, SFC, SQM. Reivindicación de derechos. www.plataformafibromialga.org

Lera Miquel, S.; Características y respuesta al tratamiento multidisciplinar de pacientes afectadas de fibromialgia. Memoria de tesis doctoral. Universitat de Barcelona. Departament de Psiquiatria i Psicobiologia clínica. Programa de doctorat: 'Recerca i clínica dels trastorns mentals'. Bienni 1998-2000.

Martínez VÁzquez, J.; ¿Cómo tratamos la fibromialgia?. Rev. Soc. Esp. Dolor 8: 511-513, 2000.

Paulino Tevar, J.; Segura Escobar, E.; Revuelta Evrard, E.; Depresión, ansiedad y fibromialgia. Rev Soc Esp Dolor 17 (2010); $7: 326$ - 332.

$<$ http://revista.sedolor.es/articulo.php?ID $=706>$

Peñacoba Puente, C. (2009).Universidad Rey Juan Carlos. Intervención psicológica en personas con fibromialgia.

Pernia Romero, A.; La fibromialgia no debe ser ese cajón de sastre. Rev Soc Esp Dolor 17 (2010); 5: $225-226$

$<$ http://revista.sedolor.es/articulo.php?ID=685> 
Ruiz, A., Ubago, M.C.; Bermejo, M.J.; Plazaola, J.; Castaño, J.; de Labry-Lima, O. y Hernández, E. Diferencias en características sociodemográficas, clínicas y psicológicas entre hombres y mujeres diagnosticados de fibromialgia. Rev Clin Esp. 2007; 207(9):433-9.

Sedó, R. y Ancochea, J. Una reflexión sobre la fibromialgia. Aten Primaria 2002, 29 (9): 562-564.

$<$ http:/www.elsevier.es/sites/default/files/elsevier/pdf/27/27v29n09a13032492pdf001.pdf>

Ubago Linares, M.C.; Ruiz Pérez, I.; Bermejo Pérez, M.J.; Olry de Labry Lima, A. y Plazaola Castaño, J. Características clínicas y psicosociales de personas con fibromialgia. Repercusión del diagnóstico sobre sus actividades. Revista Española de Salud Pública, 2005; 79: 683-695.

Valls, C. Diagnóstico diferencial del dolor y de la fibromialgia. Anuario de Psicología, 2008, vol. 39, n' 1, 87-92.

Vololona Rabeharisoa. From representation to mediation: The shaping of collective mobilization on muscular dystrophy in France. Social Science \& Medicine, 62 (2006) $564-576$.

Villanueva Vl, Valía JC, Cerdá G, Monsalve V, Bayona MJ, de Andrés J. Fibromyalgia: diagnosis and treatment. Current knowledge. Rev Soc Esp Dolor 2004; 11: 430-443. $<\mathrm{http}$ ://scielo.isciii.es/pdf/dolor/v11n7/revision2.pdf $>$

\section{2. Ámbito de la Psicología}

Bayes, R. Psicología del sufrimiento y de la muerte. Anuario de Psicologia, 1998, vol. 29, no 4,5-17.

Briones, E. (2009). Identidades femeninas en un mundo plural. Ed. Elena Jaima de Pablos. $<$ http://www.audem.com/documentos/identidadesfemeninas.pdf $>$

Comeche, M. I., Martín, A., Rodríguez, M. F., Ortega, J., Díaz, M. I. y Vallejo, M. A. Tratamiento cognitivo-conductual, protocolizado y en grupo, de la fibromialgia. Clínica y Salud, 2010; 21 (2), 107-121.

$<$ http://redalyc.uaemex.mx/redalyc/pdf/1806/180615543001.pdf>

Centro de Publicaciones del Ministerio de Sanidad y Consumo, Serie Ordenación de prestaciones, Madrid, 2003. Psicothema, 7 (1995), pp. 627-639.

De Gracia, M.; Marco, M. y Ruíz, J. (1998). Protocol mesura fibromialgia. UdG. $<$ http://www.psicolegs.org/Fotos/protocol.pdf $>$

Garcia-CAmpayo, J. y Rodero, B. Validation of a Spanish language version of the Pain Self-Perception Scale in patients with fibromyalgia. BMC Musculoskeletal Disorders 2010, 11:255. 
Gonzalez, H ; Capilla, P (2009): Detección de la simulación del dolor crónico. Un enfoque multidisciplinar.

GUITART, J. La fibromialgia y aspectos relacionados. Madrid, MAPFRE, 2000.

Martínez, C. y Paterna, C. Comprender la fibromialgia desde una perspectiva psicosocial feminista. Encuentros de Primavera, 2003; 1 (4), 100-104.

Merayo, LA, CAno, FJ, Rodríguez, L. (2008). Motivación para el cambio como predictor de la adherencia terapeútica en el dolor crónico. Apuntes de Psicología. Vol. 26 (2), pp. 331-339.

$<$ http://www.infocop.es/view_article.asp?id=2107>

Munguia-Izquierdo, D., Legaz-Arrese, A., Moliner-Urdiales, D. y Reverter-Masia, J. (2008). Neuropsicología de los pacientes con síndrome de fibromialgia. Psicothema, 20 (3), 427-431.

Munguia-Izquierdo, D., Legaz-Arrese, A. El dolor y la ansiedad se relacionan con el deterioro cognitivo en pacientes con síndrome de fibromialgia, 2008, $<$ http://www.infocop.es/view_article.asp?id=2108>

Perez-Pareja i al. (2004). Fibromialgia y emociones negativas. Psicothema. 2003, Vol 16.

Ros, N. (2006). Intervención psicológica en la fibromialgia.

$<$ http://www.infocop.es/view_article.asp?id=987>

Soucase, S., Monsalve, V.; Soriano, J.F. y De Andrés, J. Estrategias de afrontamiento ante el dolor y calidad de vida en pacientes diagnosticados de fibromialgia. Rev. Soc. Esp. Dolor, 2004,11: 353-359.

Valverde, C. y Markez, I. "Lo que tú tienes que hacer es...": El uso coercitivo de Terapia Cognitivo-Conductual en las nuevas enfermedades. Norte de Salud Mental, 2009; 35, p. $89-106$.

Valverde, C.; Markez, I. y Visiers, C. (2009). Breves intervenciones en el largo viaje: la comunicación con pacientes con fibromialgia o síndrome de fatiga crónica. OMeditorial. Colección Prometeo.

ValVerde, C. «Los cuerpos del delito»: injusticias y oportunidades en los Síndromes de Sensibilidad Central. Papeles de relaciones ecosociales y cambio global, 2010/11; $\mathrm{n}^{\mathrm{o}}$ 112, pp. 141-153.

$<$ http://www.ligasfc.org/wp-content/uploads/2011/02/los-cuerpos-del-delito_C.VALVERDE.pdf $>$

VAlLeJo, M.A. La terapia cognitivo-conductual es el tratamiento psicológico que más evidenaic aporta sobre el dolor crónico.

$<$ http://www.infocop.es/view_article.asp?id=2784>

White, Mary Terrell , Lemkau, Jeanne Parr and Clasen, Mark E. 'Fibromyalgia'. Women \& Therapy, 2001, 23: 1, $45-58$. 


\section{3. Ámbito de la Normativa y el Derecho}

ADEPACI (Asociación Civil Argentina en Defensa del Paciente). Leyes que amparan a las personas con Fibromialgia, Síndrome de Fatiga Crónica y Sensibilidad Química Múltiple. Consultada 10 Mayo 2013:

$<$ https://es-es.facebook.com/ADEPACI/posts/772292859463897>

CoL.Lectiu Ronda (2009). Las enfermedades invisibles: fibromialgia y fatiga crónica. ARA LLIBRES.

García López A; Campos Sánchez S. Impacto socioeconómico de la fibromialgia (2000). Rev. Esp. Reumatol ; 27: 447-449.

LóPEZ-TAMÉs, R (2012). La valoración incapacitante de la fibromialgia y del síndrome de fatiga crónica en el ámbito administrativo y en el judicial. Consultado 10 Mayo 2013 en: $<$ http://www.elderecho.com/laboral/valoracion-incapacitante-fibromialgiaadministrativo-judicial_11_397180007.html>

LousADA, Jose Fernando (2009). El tratamiento jurídico de la fibromialgia en perspectiva de género.

$<$ http://www.afibrosal.org/documentos/legislacion/juridicojuridicoFibr.pdf $>$

Moreno, J.V. (2013). Conferencia: Fibromialgia y síndrome de fatiga crónica. Entorno legal. Valle Hebrón. Barcelona.

Proposició de llei Fatiga crónica (2008). Consultado Febrero 2013

$<$ http://www.parlament.cat/activitat/dspcp/08p052.pdf> 